\title{
Stochastic Inverse Consistency in Medical Image Registration
}

\author{
Sai Kit Yeung ${ }^{1}$ and Pengcheng $\mathrm{Shi}^{2}$ \\ 1 Bioengineering Program \\ 2 Department of Electrical and Electronic Engineering, \\ Hong Kong University of Science and Technology, \\ Clear Water Bay, Kowloon, Hong Kong \\ \{beysk, eeship\}@ust.hk
}

\begin{abstract}
An essential goal in medical image registration is, the forward and reverse mapping matrices should be inverse to each other, i.e., inverse consistency. Conventional approaches enforce consistency in deterministic fashions, through incorporation of sub-objective cost function to impose source-destination symmetric property during the registration process. Assuming that the initial forward and reverse matching matrices have been computed and used as the inputs to our system, this paper presents a stochastic framework which yields perfect inverse consistency with the simultaneous considerations of the errors underneath the registration matrices and the imperfectness of the consistent constraint. An iterative generalized total least square (GTLS) strategy has been developed such that the inverse consistency is optimally imposed.
\end{abstract}

\section{Introduction}

One of the most desirable properties for registration is inverse consistency or source-destination symmetry in which the correspondence is one-to-one and also unambiguous. Consistent transformations maintain the topology of the registering pair. This is important in medical image registration for generating biologically meaningful results [1]. The inverse consistent constraint has been enforced with other information such as image intensity and geometric characteristics to become part of the optimization criterion in medical image registration [1] or to act as a sub-objective cost function in point set matching [3]. Since the inverse consistency in the latter case is only part of the metric which needs to be minimized, the resulting transformation matrices are, in general, not perfectly inverse consistent. Furthermore, all the above approaches solve the transformations in a deterministic nature, meaning that the stochastic properties of these matrices are not considered.

We propose a stochastic framework for registration problems which generates perfect source-destination symmetric mapping between the data sets. Instead of imposing inverse consistency in a deterministic and imperfect sense, we enforce the inverse consistent property optimally with the systematic considerations of the stochastic uncertainties of the input forward and reverse transformation matrices to achieve perfect source-destination symmetry. The adoption of the 
Generalized Total Least Square(GTLS) technique [6] allows simultaneous considerations of the errors in the input transformation matrices and the inverse consistent constraint during a post-registration fitting process to solve a set of new forward and reverse transformations iteratively until they are perfectly inverse to each other. This framework can be used with any registration algorithms which have already shown their validity in establishing forward/reverse mappings for different matching problems.

\section{Inverse Consistency in Medical Image Registration}

\subsection{Discrete Nature of the Information Sources}

Due to the discrete nature of data, either the point representation or the digital medical image of the biological objects and the discrete optimization process, correspondences extracted from conventional registration algorithms are always ambiguous, i.e., the forward and reverse mapping is not consistent. Fig.1(g) and (j) show 2 simple $1 \mathrm{D}$ examples to illustrate the point: $A_{n}$ and $B_{n}$ are discrete version of the original continuous signals $A$ and $B$ which $B$ is shifted to right by $0.5 \mathrm{~s}$ from $A=\sin (\mathrm{x}) . A_{c}$ and $B_{c}$ are the reconstructed signals for registration. Notice that $A_{c}$ and $B_{c}$ on the above examples are unable to represent the original signals perfectly due to the inadequate sampling rate of $A_{n}$ and $B_{n}$. Conventional optimization processes initialize one way to start climbing the hill (the matching criteria curve), e.g. from left to right. In Fig.1(h), the possible forward and reverse registration results would be an ambiguous pair $(-1.1,-0.5)$ instead of the ground truth pair $(-0.5,0.5)$. In Fig.1(k), the matching criteria curves give a consistent pair $(-1,1)$, however, it is not the ground truth transformation 1 .

\subsection{Deterministic Inverse Consistent Constraint}

One typical scheme to incorporate inverse consistency for registration is to assign a cost metric $E_{C o n s}$ for the inverse consistent property as part of the matching cost function $E$, i.e., $E=E_{\text {Sim }}+E_{\text {Cons }}$ where $E_{\text {Sim }}$ measures the similarity (i.e. image intensity and geometrical properties) between the data sets. Since the consistency is only part of the overall cost function, the optimal solution to Equ. 1 in general would not produce the perfect source-destination symmetry one desires.

Moreover, this type of formulation didn't consider the underlying stochastic uncertainties such that the forward transformation $T_{12}$ and the reverse transformation $T_{21}$ are solved in deterministic nature in order to get a one-to-one consistent mapping (consistent correspondence), i.e.,

$$
T_{12} * T_{21}=I \text {. }
$$

\footnotetext{
${ }^{1}$ It should be noticed that inverse consistency can always be automatically achieved if the registering pair is continuous (Fig.1(a),(b)) or the digital signal is sampled under very high sampling rate such that the original continuous signal can be perfectly reconstructed (Fig.1(d),(e)).
} 


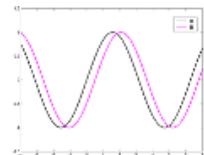

(a)

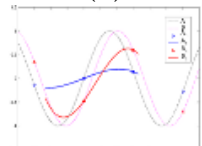

(g)

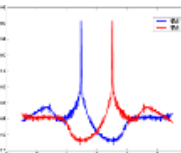

(b)

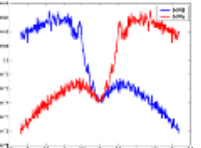

(h)

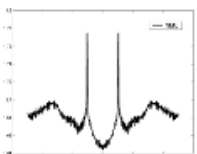

(c)

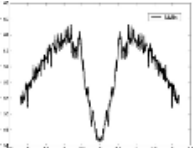

(i)

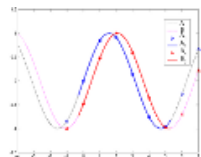

(d)

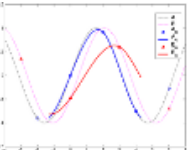

(j)

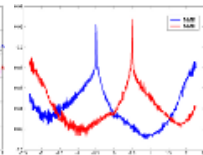

(e)

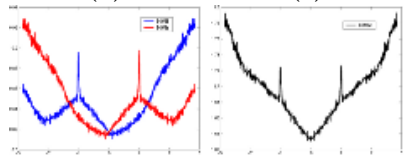

(k)

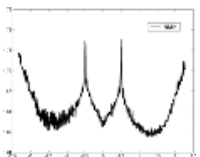

(f)

(l)

Fig. 1. Column 1 and $4: A_{c}$ and $B_{c}$ are the registering pair reconstructed from $A_{n}$ and $B_{n}$ which are the sampled version of $A$ and $B$ respectively (in (a): $A_{c}=\mathrm{A}, B_{c}=\mathrm{B}$ ). Column 2 and 5: the blue curve (NMIf) shows the forward matching criteria (registering $B_{c}$ to $A_{c}$ ) while the red one (NMIr) for registering $A_{c}$ to $B_{c}$. Column 3 and 6: The combined matching criteria curve (NMIc) from the forward and reverse registration process, here the combination is simple addition. NMI is the normalized mutual information [5].

\subsection{Role of Inverse Consistency in Registration}

In one dimensional, imposing inverse consistency deterministically means the hill climbing process should be in pairwise nature: $(1,-1) \ldots(8,-8)$ for the testing signal over the reference signal. Equivalently, there would be a new matching criteria curve that is a combination of the forward and reverse matching criteria curve. The simplest way is to have a non-weighted linear combination [4, which is equivalently a simple addition, as shown column 3 and 6 in Fig.1. Here, a critical rule for combining the forward and reverse matching criteria curves under a deterministic sense is that they should be combined in the corresponding transformation position, i.e. the NMIf value at 0.5 translation have to combine with the NMIr value at 0.5 translation also. In fact, deterministic consistency will only give better registration results if a new peak closer to the ground truth is formed as shown in Fig.1(i), which the transformation pair corresponding to optimum will be around $(-0.8,0.8)$ instead of $(-1.1,-0.5)$ and also closer to the ground truth $(-0.5,0.5)$.

\section{Stochastic Inverse Consistency in Medical Image Registration}

\subsection{Stochastic Inverse Consistent Constraint}

As we have stated above, the discrete nature of the information source makes the matching criteria drive to incorrect maximum, simply combine them deterministically will not be an optimal way to utilize the information from the forward and reverse process. In this paper, we are arguing that one should model inverse consistency stochastically with the simultaneous consideration of the underlying stochastic uncertainties within the forward and reverse transformation matrices and hence the imperfectness of the inverse consistent constraint, i.e, 


$$
\begin{aligned}
\left(T_{12}+E_{T_{12}}\right) *\left(T_{21}+E_{T_{21}}\right) & =I+R_{i} \\
E_{T_{12}}=\left|T_{12}-T_{21}^{-1}\right| & E_{T_{12}^{-1}}
\end{aligned}
$$

We adopt a simple absolute difference approach for $E_{T_{12}}$ and $E_{T_{21}}$ ( $E_{T_{21}}$ is obtained from $E_{T_{12}^{-1}}$, which will be explained later) to model the stochastic error properties of the transformation matrix $T_{12}$ and $T_{21} 2$ respectively since the forward and inverse of the reverse transformation has already set up a loose upper bound of the error. $R_{i}$ is the error imposed on the imperfectness of the inverse consistent constraint 3 . Under this formulation, we can provide more flexibility on imposing source-destination symmetry between the forward and reverse registration processes, without compromising accuracy.

To further simplify our current error model, we assume all the elements in the error matrices have zero mean and are independent to each other. These matrices will be involved in building the error equilibration matrices for the Generalized Total Least Solvers in the following section.

\subsection{GTLS Formulation}

As the stochastic property are not the same for every entry and some of the entries are error free, in order to solve the problem while considering all the errors simultaneously, a Generalized Total Least Square (GTLS) 6] approach is adopted. Consider a overdetermined system of linear equations

$$
A X \approx B \quad A \in R^{m \times n}, B \in R^{m \times d} \text { and } X \in R^{n \times d}, m \geqslant n+d
$$

If the first $n_{1}$ column in $A$ is error free, $A$ can be partitioned into $\left[A_{1}, A_{2}\right]$ where $A_{1} \in R^{m \times n_{1}}, A_{2} \in R^{m \times n_{2}}$ and $\mathrm{n}=n_{1}+n_{2}$. A GTLS solution of (4) is any solution of the set $\widehat{A} X=A_{1} X_{1}+\widehat{A_{2}} X_{2}=\widehat{B} . \widehat{A}=\left[A_{1}, \widehat{A_{2}}\right]$ and $\widehat{B}$ are determined such that Range $(\widehat{B}) \subseteq \operatorname{Range}(\widehat{A})$ and $\left\|R_{D}^{-T}\left[\triangle \widehat{A_{2}}, \triangle \widehat{B}\right] R_{C}^{-1}\right\|_{F}=\| R_{D}^{-T}\left[A_{2}-\right.$ $\left.\widehat{A_{2}}, B_{2}-\widehat{B}\right] R_{C}^{-1} \|_{F}$ is minimal where $R_{D} \in R^{m \times m}$ and $R_{C} \in R^{\left(n_{2}+d\right) \times\left(n_{2}+d\right)}$ are the given equilibration matrices such that the errors on $R_{D}^{-T}\left[A_{2}, B\right] R_{C}^{-1}$ are equilibrated, i.e. uncorrelated with zero mean and same variance.

Our objective is to solve the fitting transformation matrices under the consideration of the errors in the transformation matrices and the source-destination symmetric constraint simultaneously by making use of the GTLS property. Notice that the last row of the affine transformation matrix is actually error free. By making use of this property, the transformation matrices can be first transposed and permuted to fit the GTLS formulation:

$$
\begin{aligned}
Q_{12} & =T_{12}^{T} * P & Q_{21} & =T_{21}^{T} * P \\
\text { inv } Q_{12} & =\left(T_{12}^{-1}\right)^{T} * P & \text { inv } Q_{21} & =\left(T_{21}^{-1}\right)^{T} * P
\end{aligned}
$$

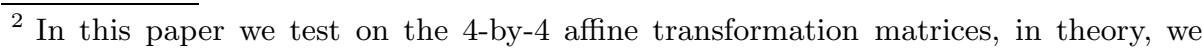
can also enforce the stochastic relationship on non-rigid deformation. Notice that we didn't apply our model on rigid transformation due to the orthonormality issue.

${ }^{3}$ We simply assume all the entries in $R_{i}$ have the same stochastic uncertainty and set it as $\triangle_{r}$, i.e., $R_{i} \in R^{4 \times 4}$ with all the entries equal to $\triangle_{r}$.
} 


$$
P=P_{14} * P_{24} * P_{34}, P_{14}=\left(\begin{array}{cccc}
0 & 0 & 0 & 1 \\
0 & 1 & 0 & 0 \\
0 & 0 & 1 & 0 \\
1 & 0 & 0 & 0
\end{array}\right) \quad P_{24}=\left(\begin{array}{cccc}
1 & 0 & 0 & 0 \\
0 & 0 & 0 & 1 \\
0 & 0 & 1 & 0 \\
0 & 1 & 0 & 0
\end{array}\right) \quad P_{34}=\left(\begin{array}{llll}
1 & 0 & 0 & 0 \\
0 & 1 & 0 & 0 \\
0 & 0 & 0 & 1 \\
0 & 0 & 1 & 0
\end{array}\right)
$$

$Q_{12}$ will be a 4 -by-4 matrix with the form:

$$
Q_{12}=\left(\begin{array}{cccc}
0 & T_{12}(1,1) & \cdots & T_{12}(3,1) \\
0 & T_{12}(1,2) & & \vdots \\
0 & \vdots & \ddots & \vdots \\
1 & \vdots & \cdots & T_{12}(3,4)
\end{array}\right) \quad Q_{21}=\left(\begin{array}{cccc}
0 & T_{21}(1,1) & \cdots & T_{21}(3,1) \\
0 & T_{21}(1,2) & & \vdots \\
0 & \vdots & \ddots & \vdots \\
1 & \vdots & \cdots & T_{21}(3,4)
\end{array}\right)
$$

So the first column of $Q_{12}$ and $Q_{21}$ is error free and suit the form of the GTLS approach stated before. Hence the GTLS formulation of our stochastic inverse consistent model becomes:

$$
\left[\begin{array}{c}
Q_{12} \\
i n v Q_{21}
\end{array}\right] X \approx\left[\begin{array}{l}
I \\
I
\end{array}\right] \quad\left[\begin{array}{c}
i n v Q_{12} \\
Q_{21}
\end{array}\right] Y \approx\left[\begin{array}{l}
I \\
I
\end{array}\right]
$$

The optimal forward and reverse transformation $T_{12}^{*}$ and $T_{21}^{*}$ are obtained by performing the permutation and transpose on the GTLS solutions $X$ and $Y$ :

$$
T_{21}^{*}=(P * X)^{T} \quad T_{12}^{*}=(P * Y)^{T}
$$

Apart from the input transformation matrices, the error properties are also necessary to specify the GTLS formulation. The error matrices $E_{Q_{12}}, E_{i n v Q_{12}}$ for $Q_{12}$, inv $Q_{12}$ are derived as the same in Equ.(3) i.e.,

$$
E_{Q_{12}}=\left|Q_{12}-i n v Q_{21}\right| \quad E_{i n v Q_{12}}=\left|Q_{21}-i n v Q_{12}\right|
$$

and the first column is dropped as the first column of $Q_{12}$ is error free. The error matrices $E_{i n v Q_{21}}$ and $E_{Q_{21}}$ transformation matrix are formed respectively by:

$$
E_{i n v Q_{21}}=\frac{(1-\alpha)}{\alpha} * E_{Q_{12}} \quad E_{Q_{21}}=\frac{(1-\alpha)}{\alpha} * E_{i n v Q_{12}}
$$

where $\alpha$ is the weighting on the error of the forward transformation matrix $T_{12}$ :

$$
\alpha=\frac{\text { voxel size of } I_{1}}{\text { voxel size of } I_{2}} \text { or } \alpha=\frac{\# \text { of points in point set } 2}{\# \text { of points in point set } 1}
$$

So by imposing the above relationship, the registration result with a higher resolution testing image or point matching result with more points in the testing point set will be trusted more. While in this paper we use this simple assumption to model the weighting function between the error on forward and reverse registration results from two images under different resolutions. More complicated way can be investigated and would be one possibility of our future work.

The error equilibration matrices $R_{C}$ and $R_{D}$ are obtained from the Cholesky decomposition of the error covariance matrices $\mathrm{C}$ and $\mathrm{D}$, where $C=\Delta^{T} \Delta$, $D=\Delta \Delta^{T}, \Delta=\left[\begin{array}{cc}E_{Q_{12}} & R_{i} \\ E_{i n v Q_{21}} & R_{i}\end{array}\right] . \Delta$ represents the stochastic property of the error in solving $X$ in Equ.(9), while $\Delta$ matrix in solving $Y$ is $\left[\begin{array}{cc}E_{i n v Q_{12}} & R_{i} \\ E_{Q_{21}} & R_{i}\end{array}\right]$. 


\subsection{Inverse Consistency by Iterative GTLS Solution}

After defining the GTLS model for fitting the transformation matrix based on our stochastic source-destination symmetric model, we set up the whole iterative process from the registration results $T_{12}$ and $T_{21}$ in order to extract both the forward transformation matrix $T_{12}^{*}$ and the reverse transformation matrix $T_{21}^{*}$ which are inverse of each other. The input for the iteration process is $Q_{12}, Q_{21}$, $i n v Q_{12}$, inv $Q_{21}$ in Equ.(5) and (6).

$$
\left[\begin{array}{c}
Q_{12}^{(0)} \\
i n v Q_{21}^{(0)}
\end{array}\right] X^{(0)} \approx\left[\begin{array}{l}
I \\
I
\end{array}\right]\left[\begin{array}{c}
i n v Q_{12}^{(0)} \\
Q_{21}^{(0)}
\end{array}\right] Y^{(0)} \approx\left[\begin{array}{l}
I \\
I
\end{array}\right]
$$

with the corresponding stochastic property in the noise data:

$$
\left[\begin{array}{cc}
E_{Q_{12}}^{(0)} & R_{i} \\
E_{\text {inv }}^{(0)} & R_{i}
\end{array}\right] \quad \text { and } \quad\left[\begin{array}{cc}
E_{\text {inv }}^{(0)} & R_{i} \\
E_{Q_{21}}^{(0)} & R_{i}
\end{array}\right]
$$

the ' 0 ' in the brackets is the number of iteration and the solved $X^{(0)}$ and $Y^{(0)}$ are:

$$
\begin{array}{ccc} 
& X^{(0)}=P^{-1} *\left(T_{21}^{(1)}\right)^{T} & \multicolumn{2}{c}{Y^{(0)}=P^{-1} *\left(T_{12}^{(1)}\right)^{T}} \\
\text { so } \quad\left(X^{(0)}\right)^{-1}=\operatorname{inv} Q_{21}^{(1)} \quad & \text { and } \quad P *\left(X^{(0)}\right) * P=Q_{21}^{(1)} \\
& \left(Y^{(0)}\right)^{-1}=\operatorname{inv} Q_{12}^{(1)} \quad \text { and } & P *\left(Y^{(0)}\right) * P=Q_{12}^{(1)}
\end{array}
$$

The corresponding error matrices for the transformation matrices are also updated during the iteration, i.e., getting $E_{Q_{12}}^{(1)}, E_{Q_{21}}^{(1)}, E_{i n v Q_{12}}^{(1)}, E_{i n v Q_{21}}^{(1)}$ by Equ.(11) and (12) to fit the input matrices of the GTLS solvers as the transformation errors should be smaller during the iteration (closer to the ground truth) while the error matrix $R_{i}$ for the source-destination symmetric constraint is fixed as the initial input stochastic consistent model is kept unchanged. So all the components for the GTLS solvers are updated and the process can be repeated until

$$
\left\|\left(P * X^{(n)}\right)^{T} *\left(P * Y^{(n)}\right)^{T}-I\right\|_{F}<\text { threshold }
$$

and the GTLS solution matrices will be:

$$
T_{21}^{*}=\left(P * X^{(n)}\right)^{T} \quad T_{12}^{*}=\left(P * Y^{(n)}\right)^{T}
$$

\section{Experiments and Discussion}

We have applied our stochastic inverse consistent model on registration of point sets which representing the human brain in Fig.2. Feature points are selected from the brain image to act as the testing point set, then a non-rigid mapping, Gaussian radial basis functions was applied on it to form the reference point set. Different degree of gaussian noise and different proportion of outliers are added to both point sets in the experiment. The Robust point matching algorithm 


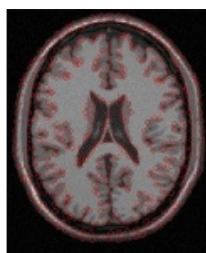

(a)

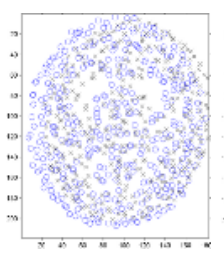

(b)

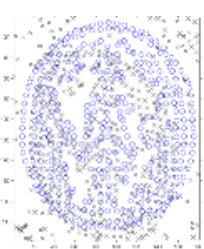

(c)

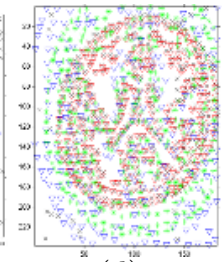

(d)

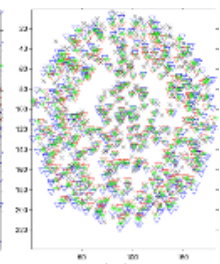

(e)
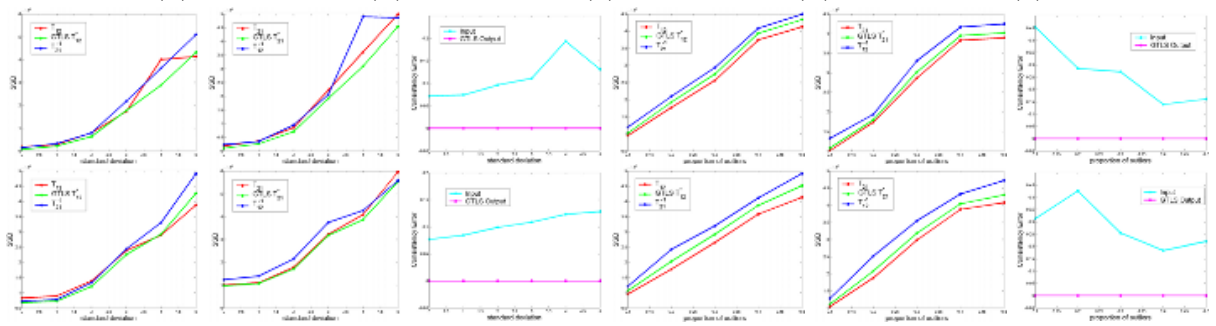

Fig. 2. (a):Brain image with the extracted point set (the testing point set). (b),(c): Testing point sets (blue circle) and the reference point sets (black cross). (b) small deformation, noise level $=2 \mathrm{SD}$. (c) large deformation, outlier proportion $=0.5$. $(\mathrm{d})$ :Forward wrapping results: small deformation, outlier proportion $=0.1$. (e):Reverse wrapping results: large deformation, noise level $=4 \mathrm{SD}$. Color convention for all the results shown in the figure: forward process - red: $T_{12}$, green: $T_{12}^{*}$, blue: $T_{21}^{-1}$, reverse process - red: $T_{21}$, green: $T_{21}^{*}$, blue: $T_{12}^{-1}$. The 2 nd and 3rd row are the results for small deformation and large deformation respectively. Column 1 to 3 are the results for different noise level, column 4 to 6 are for different proportion of outliers. Column 1 and 4 are the errors computed as sum of squared distance (SSD) between the points in the warped testing point set and the reference point set for forward process while column 2 and 5 are for reverse process. Column 3 and 6 are the consistency error computed as $\left\|T_{12} * T_{21}-I\right\|_{F}$ for the input and $\left\|T_{12}^{*} * T_{21}^{*}-I\right\|_{F}$ for the GTLS output.

RPM [2] is run on the pair of point sets to obtain the forward and reverse transformation matrices for our system. The position error of the points is computed as the sum of squared distance (SSD) between the points in the warped testing point set and the reference point set for the evaluation of the transformations obtained. We also compare the error on consistency by $\left\|T_{12} * T_{21}-I\right\|_{F}$. As it is expected, our stochastic inverse consistent model generates a perfect sourcedestination symmetric registration results from the input forward and reverse transformation matrices which are inconsistent in nature.

Moreover, our GTLS solutions will produce results those will always better than the worst and sometimes be the best as shown in column 1 and 2 in Fig.2. Actually proper modelling of individual element of the error matrices, their relationship within the matrix and also the interrelation among the error matrices will be the potential mean to improve the registration results through consistency so that the GTLS solutions always yield the best results. This modelling is depended on the actual data and also the corresponding matching criteria 

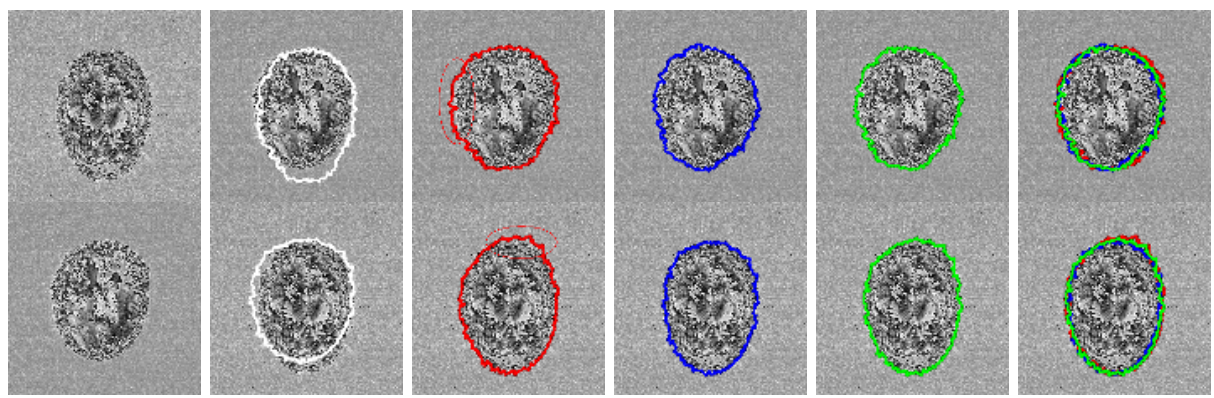

Fig. 3. Column 1: two different PD-weighted MRIs. Row 1 and 2 are the forward and reverse registration results. Forward results - red: $T_{12}$, green: $T_{12}^{*}$, blue: $T_{21}^{-1}$, reverse results - red: $T_{21}$, green: $T_{21}^{*}$, blue: $T_{12}^{-1}$. White: contour of the unregistered testing image overlay the reference image.

which is very complicated and will be investigated in our future work. In real registration problem, ground truth is not available and the complicated input image or point data make it very difficult to determine the forward or reverse transformation is superior than the other. Hence our stochastic inverse consistent model can always produce better result in terms of robustness. Fig.3 shows the registration results for $2 \mathrm{PD}$-weighted MRIs. The inconsistency of the forward and reverse process is shown in the figures by the red and blue contours. $T_{12}^{*}$ and $T_{21}^{*}$ is in-between their inputs and also perfectly inverse to each other. In addition, the observable registration errors in $T_{12}$ and $T_{21}$ from the red contours are not appeared in our GTLS solutions which show that the stochastic model produce better registrations.

\section{Conclusion}

We presented a novel framework for modelling inverse consistency stochastically, by simultaneously considering the stochastic uncertainties on both of the transformation matrices and the source-destination symmetric constraint through the Generalized Total Least square fitting from the transformation matrices obtained after the registration process. With our stochastic inverse consistent model, source-destination symmetry can be enforced perfectly with the consideration of any other similarity constraints. This work is supported by HKRGC CERG Grant HKUST6151/03E.

\section{References}

1. Gary E. Christensen and Hans J. Johnson. Consistent image registration. IEEE Trans. Med. Imaging, 20(7):568-582, 2001.

2. Haili Chui and Anand Rangarajan. A new algorithm for non-rigid point matching. In $C V P R$, pages 2044-2051, 2000. 
3. Hongyu Guo, Anand Rangarajan, S. Joshi, and Laurent Younes. Non-rigid registration of shapes via diffeomorphic point matching. In ISBI, pages 924-927, 2004.

4. Oskar M. Skrinjar and Hemant Tagare. Symmetric, transitive, geometric deformation and intensity variation invariant nonrigid image registration. In $I S B I$, pages 920-923, 2004.

5. Colin Studholme, Derek L. G. Hill, and David J. Hawkes. An overlap invariant entropy measure of 3d medical image alignment. Pattern Recognition, 32(1):71-86, 1999.

6. S. Van Huffel and J. Vandewalle. Analysis and properties of the generalized total least squares problem $A X \approx B$ when some or all columns in $A$ are subject to error. SIAM J. Matrix. Anal. Appl., 10:294-315, 1989. 